\title{
Current Status of Lung Fluke Metacercarial Infection in Freshwater Crabs in the Kawane Area of Shizuoka Prefecture, Japan
}

\author{
Hiromu SUGIYAMA $^{1) *}$, Katsumasa SHIBATA ${ }^{1)}$, Yasuyuki MORISHIMA ${ }^{1)}$, Maki MUTO ${ }^{1)}$, Hiroshi YAMASAKI ${ }^{1)}$ and \\ Yasushi KAWAKAMI ${ }^{2}$ \\ 1) Department of Parasitology, National Institute of Infectious Diseases, Toyama 1-23-1, Shinjuku-ku, Tokyo 162-8640, Japan \\ ${ }^{2)}$ Laboratory of Parasitology, School of Life and Environmental Science, Azabu University, 1-17-71 Fuchinobe, Chuo-ku, Sagamihara, \\ Kanagawa 229-8501, Japan
}

(Received 20 July 2012/Accepted 1 October 2012/Published online in J-STAGE 18 October 2012)

\begin{abstract}
Feline cases of lung fluke infection were recently reported in the upper basin of the Oi River in Shizuoka Prefecture. The causative species of these cases were not identified, although a field survey conducted about 40 years ago in this area demonstrated the prevalence of Paragonimus miyazakii metacercariae in intermediate host crabs. To clarify the current status of lung fluke metacercarial infection in host crabs, we collected the Japanese freshwater crab or Sawagani, Geothelphusa dehaani, at 14 sampling sites in the Kawane area, which is located in the upper basin of the Oi River. Sawagani harboring Paragonimus metacercariae were collected at all sampling sites in this study with a total prevalence of $42 \%$ (281 of $677 \mathrm{crabs}$ examined) and a range of $16 \%$ to $92 \%$. The number of metacercariae per infected crab ranged from 1 to 19 with a mean of 2.2. Based on the morphological features of the metacercariae and adult worms recovered from rats that were experimentally infected with the metacercariae, the lung flukes examined were identified as $P$. miyazakii Kamo, Nishida, Hatsushika and Tomimura, 1961; ITS2 sequence data corroborate this conclusion. P. miyazakii is still widely prevalent in this area, implying that the recently reported feline paragonimiasis cases were likely caused by $P$. miyazakii infection.

KEY wORDS: Japanese freshwater crab, lung fluke, Paragonimus miyazakii, Paragonimus skrjabini, Sawagani.
\end{abstract}

doi: 10.1292/jvms.12-0325; J. Vet. Med. Sci. 75(3): 249-253, 2013

Lung flukes Paragonimus westermani, Paragonimus miyazakii, and Paragonimus ohirai occur in Japan; the first 2 species are zoonotic pathogens that cause pulmonary and sometimes extrapulmonary diseases [8]. These 2 lung fluke species share a major infection source, the Japanese freshwater crab or Sawagani (sawa means mountain stream and gani [or kani] means crab in Japanese), Geothelphusa dehaani. Sawagani has a geographical distribution that covers almost all of Japan except for Hokkaido and the southern islands of Okinawa Prefecture, and Sawagani harboring metacercariae of these 2 Paragonimus spp. have been identified in several areas throughout this geographical range [9]. Among the regions in Japan to which Sawagani is endemic, the Kawane area in the upper basin of the Oi River (now merged with Shimada City) of Shizuoka Prefecture received marked attention about 40 years ago when outbreaks of human paragonimiasis in Tokyo, Kanagawa and Yamanashi prefectures were traced to the consumption of raw Sawagani harvested in Shizuoka Prefecture, particularly the Kawane area [20]. Although the patient sputum and/or fecal samples were negative for lung fluke eggs, serological tests suggested infection with $P$. miyazakii $[6,20]$. Extensive field surveys conducted throughout Shizuoka Prefecture revealed a high prevalence of $P$. miyazakii metacercariae in Sawagani

*Correspondence to: Sugiyama, H., Department of Parasitology, National Institute of Infectious Diseases, Toyama 1-23-1, Shinjuku-ku, Tokyo 162-8640, Japan.

e-mail: hsugi@nih.go.jp

(C)2013 The Japanese Society of Veterinary Science in regions along the Oi River, including in the Kawane area, but lower or zero prevalence in regions along rivers other than the Oi River [4]. Recently, paragonimiasis cases were reported in cats kept by villagers in the upper basin of the Oi River [11], but the causative species was not identified.

Here, we examined the current status of lung fluke metacercarial infection in Sawagani at 14 sampling sites in the Kawane area. These data will be useful for establishing countermeasures needed to control lung fluke infections not only in the Kawane area, but also in Tokyo, because Sawagani from several prefectures, including Shizuoka, are sold as foodstuffs at retail fish markets in Tokyo, and some were found to be positive for $P$. westermani and/or $P$. miyazakii metacercariae $[17,19]$.

\section{MATERIALS AND METHODS}

Isolation and examination of Paragonimus metacercariae: Between October 2010 and May 2012, we collected 677 Sawagani from 14 sampling sites in mountain streams in the Kawane area of Shizuoka Prefecture (Fig. 1). Stream names were assigned as sampling site names. Sawagani were examined one by one to gather accurate data on the prevalence and burden of Paragonimus metacercariae, as described previously [15]. Briefly, the internal organs including the gills, heart, midgut gland, and genital organs were excised from crabs after removing the carapace, compressed between 2 glass plates, and examined for the presence of metacercariae under a stereoscopic microscope. When Paragonimus metacercariae were identified, the glass plate covering the tissues was carefully removed and metacercariae in the compressed 
tissues were recovered using teasing needles or tweezers. Isolated metacercariae were placed on glass slides and pressed lightly under a coverslip for morphological observation and measurement.

Adult worms from test rats: Male Wistar rats ( $\mathrm{n}=2,5$-weekold) were orally infected with 21 and 33 metacercariae from 2 different sampling sites. Necropsy was performed 69 and 81 days after infection, and worms were recovered from the lungs and pleural cavity by macroscopic examination. The recovered worms were compressed between 2 glass slides, fixed in $70 \%$ alcohol, stained with borax carmine,

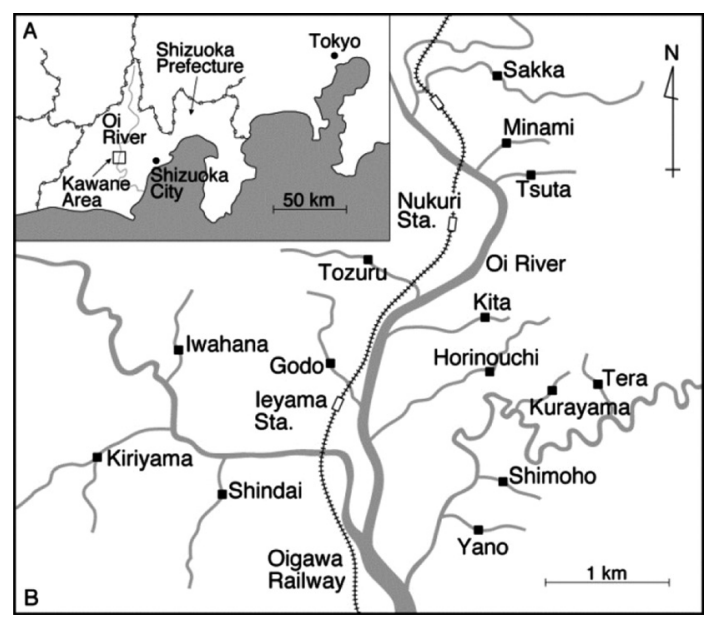

Fig. 1. Study area and sites of freshwater crab sampling.

(A) Map of central Japan showing the study area in Kawane, central Shizuoka Prefecture.

(B) Detailed map of the study area, showing sampling sites in mountain streams at which freshwater crabs, Geothelphusa dehaani, were collected. and mounted with Canada balsam for morphological observations and measurements. Developmental stages of the recovered worms were determined according to the criterion of Shibahara [13].

DNA amplification and sequencing of the second internal transcribed spacer (ITS2) region of ribosomal DNA: The ITS2 region of 2 metacercariae from each sampling site was amplified by PCR and sequenced as described previously [16]. The primers used were 3S, 5'-GGTACCGGTGGATCACTCGGCTCGTG-3' (forward), and A28, 5'-GGGATCCTGGTTAGTTTCTTTTCCTCCGC-3' (reverse). Sequences were aligned and compared using the GENETYX-Win software (Ver. 10, Genetyx Co., Tokyo, Japan).

\section{RESULTS}

Prevalence and burden of Paragonimus metacercariae in Sawagani: We collected 677 Sawagani from 14 sampling sites in the Kawane area (Fig. 1). At all examined sites, Sawagani harboring Paragonimus metacercariae were detected. The total prevalence of Paragonimus metacercariae over the entire Kawane area was $42 \%$ (281 of 677 crabs examined), ranging from $16 \%$ to $92 \%$ among sites (Table 1). A total of 631 metacercariae were collected from the 281 positive crabs, and the number collected from a single infected crab ranged from 1 to 19 (mean, 2.2 for infected crabs). Taken over all crabs collected, the mean number of metacercariae per individual was 0.93. Among sampling sites, the highest incidence (92\%) of metacercarial infection and greatest mean metacercarial burden per infected crab (4.0) were found at Yano.

Morphology of metacercariae collected from Sawagani: Paragonimus metacercariae obtained from Sawagani were spherical with thin outer and thick inner walls (Fig. 2). The

Table 1. Prevalence and burden of Paragonimus metacercariae in freshwater crabs collected from mountain streams in 14 locations in the Kawane area, Shizuoka Prefecture

\begin{tabular}{|c|c|c|c|c|c|c|}
\hline \multirow{2}{*}{ Location } & \multicolumn{2}{|c|}{ No. of crabs } & \multirow{2}{*}{$\%$ infected } & \multirow{2}{*}{$\begin{array}{l}\text { Total no. of } \\
\mathrm{mc}^{\mathrm{a})} \text { detected }\end{array}$} & \multicolumn{2}{|c|}{ No. of mc per infected crab } \\
\hline & Examined & Infected & & & Range & Average \\
\hline Sakka & 81 & 20 & 25 & 57 & $1-19$ & 2.9 \\
\hline Minami & 51 & 27 & 53 & 53 & $1-11$ & 2.0 \\
\hline Tsuta & 54 & 36 & 67 & 63 & $1-11$ & 1.8 \\
\hline Kita & 35 & 6 & 17 & 17 & $1-3$ & 2.8 \\
\hline Horinouchi & 69 & 11 & 16 & 16 & $1-2$ & 1.5 \\
\hline Tera & 55 & 33 & 60 & 60 & $1-10$ & 1.8 \\
\hline Kurayama & 32 & 11 & 34 & 34 & $1-4$ & 3.1 \\
\hline Shimoho & 48 & 26 & 54 & 54 & $1-10$ & 2.1 \\
\hline Yano & 25 & 23 & 92 & 92 & $1-10$ & 4.0 \\
\hline Shindai & 46 & 12 & 26 & 26 & $1-3$ & 2.2 \\
\hline Kiriyama & 61 & 33 & 54 & 58 & $1-11$ & 1.8 \\
\hline Iwahana & 54 & 21 & 39 & 39 & $1-4$ & 1.9 \\
\hline Godo & 25 & 8 & 32 & 32 & $1-3$ & 4.0 \\
\hline Tozuru & 41 & 14 & 34 & 30 & $1-7$ & 2.1 \\
\hline Total & 677 & 281 & 42 & 631 & - & 2.2 \\
\hline
\end{tabular}

a) mc: metacercariae. 


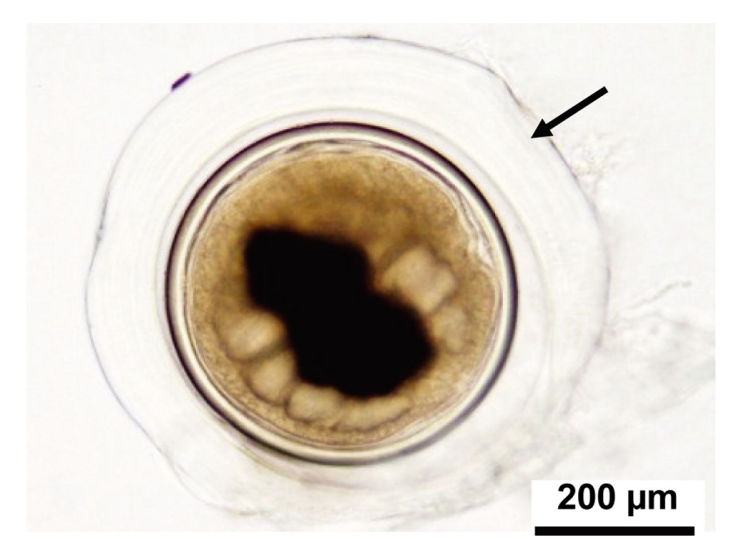

Fig. 2. Photomicrograph of a Paragonimus metacercaria. Note the very thick outermost membranous substance (arrow).

thickness of the outer cyst wall of 47 specimens from Yano ranged from 2.1 to $3.7 \mu \mathrm{m}$ (mean, $2.6 \mu \mathrm{m}$ ); that of the inner cyst wall ranged from 10.0 to $22.9 \mu \mathrm{m}$ (mean, $17.6 \mu \mathrm{m}$ ). Furthermore, the outer cyst of many specimens was tightly enveloped with a very thick membranous substance with a thickness ranging from 11.8 to $69.8 \mu \mathrm{m}$ (mean, $30.6 \mu \mathrm{m}$ ). The diameter of the inner cyst after being freed from the outer cyst ranged from 398 to $515 \mu \mathrm{m}$ (mean, $458 \mu \mathrm{m}$ ). Examination of 10 larvae showed that none had a stylet in the oral sucker.

Infection of rats with metacercariae and morphology of adult worms: Necropsy was performed on the two rats 69 and 81 days after inoculation with 21 and 33 Paragonimus metacercariae, respectively, collected from 2 different sampling sites in the Kawane area (Table 2). In Rat 1, 6 and 2 worms in the lungs and pleural cavity were identified as adults and pre-adults, respectively. Rat 2 had 10 worms in each of the lungs and pleural cavity of which 7 worms from the lungs and 2 worms from the pleural cavity were identified as adults. The remaining 11 worms were identified as pre-adults. The mean size of the 15 adult worms from the 2 rats that were mounted on glass slides was $5.8 \times 2.7 \mathrm{~mm}$, ranging from 4.4 to $6.6 \mathrm{~mm}$ in length and 2.2 to $3.3 \mathrm{~mm}$ in width. The ratio of body length to width ranged from 1.9 to 2.4 (mean, 2.1). The mean transverse diameter of the oral and ventral suckers was 540 and $680 \mu \mathrm{m}$, respectively.

A representative specimen is shown in Fig. 3, and the morphology of the adults is described as follows. The ventral sucker is always larger than the oral sucker and is situated slightly anterior to the middle of the body. The ovary is intricately branched, having a coral-like appearance (Fig. $3 \mathrm{~A})$. The uterus is situated opposite the ovary. The testes are situated on both sides of the posterior part of the body, and these are larger than the ovary. The cuticular spines are singly spaced (Fig. 3B).

ITS 2 sequence analysis: The alignment of ribosomal DNA ITS2 sequences of 28 metacercariae ( 2 from each of 14 collection sites) was $361 \mathrm{bp}$ in length and showed no variation in length or composition among the specimens. The nucleo-

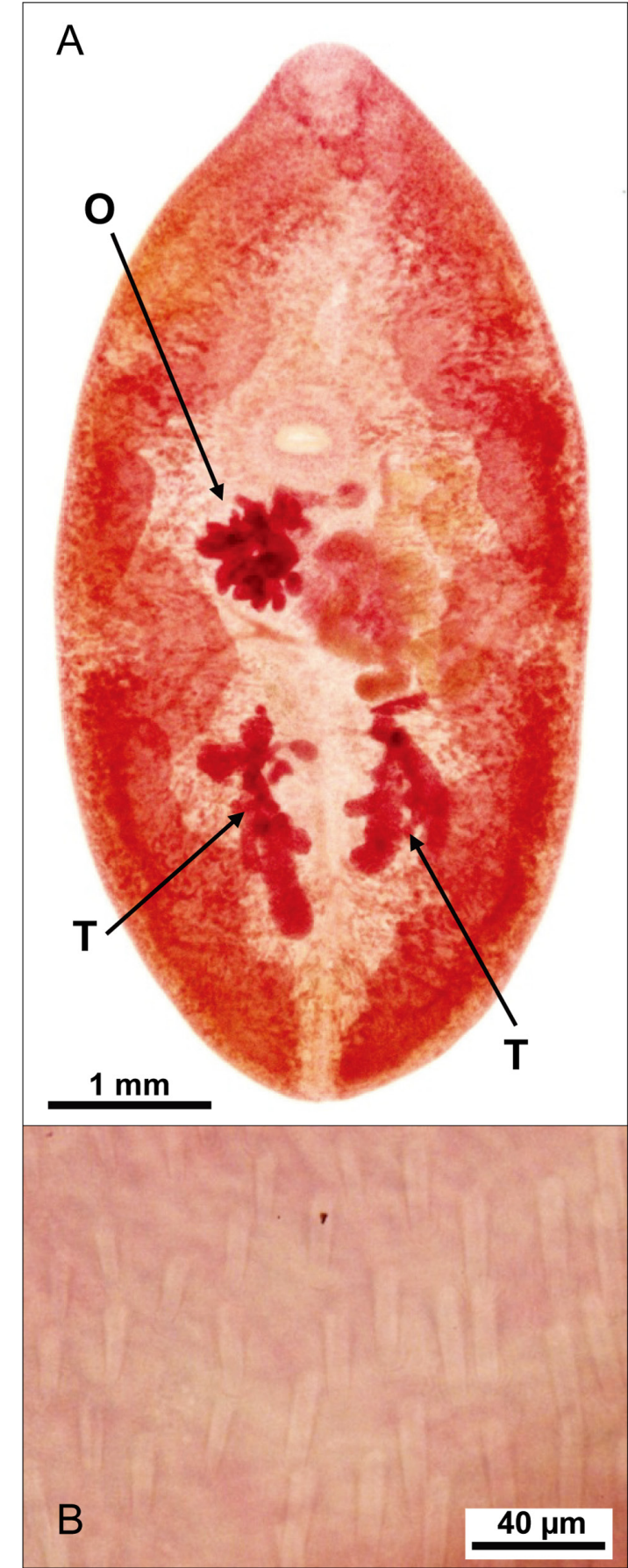

Fig. 3. Photomicrograph of an adult worm.

(A) Dorsal view of an adult worm isolated from the lung of a rat on necropsy 81 days after infection. Stained with borax carmine, showing the testes $(\mathrm{T})$ and ovary $(\mathrm{O})$.

(B) Photomicrograph of cuticular spines. Note the singly spaced arrangement.

tide sequence was deposited in the DDBJ/EMBL/GenBank database under accession number AB733177.

Searches of nucleotide databases revealed that the ITS2 
Table 2. Experimental infection of rats with Paragonimus metacercariae isolated from freshwater crabs

\begin{tabular}{|c|c|c|c|c|c|c|c|}
\hline \multirow[b]{2}{*}{ Rat No. } & \multirow[b]{2}{*}{ Source of $\mathrm{mc}^{\mathrm{a}), \mathrm{b})}$} & \multirow[b]{2}{*}{ Dose of mc } & \multirow{2}{*}{$\begin{array}{c}\text { Duration of } \\
\text { infection (days) }\end{array}$} & \multirow{2}{*}{$\begin{array}{c}\text { Rate of mc } \\
\text { recovered as (pre) } \\
\text { adult }{ }^{c)} \text { worms (\%) }\end{array}$} & \multicolumn{3}{|c|}{ No. of (pre)adult worms recovered } \\
\hline & & & & & Total & $\begin{array}{c}\text { Pleural } \\
\text { cavity }\end{array}$ & Lungs \\
\hline 1 & Tsuta & 21 & 69 & 38 & 8 & 2 & 6 \\
\hline 2 & Yano & 33 & 81 & 61 & 20 & 10 & 10 \\
\hline
\end{tabular}

a) Locations in the Kawane area from where the infected freshwater crabs were collected.

b) mc: metacercariae.

c) (pre)adult: preadult and adult.

sequence determined in this study was identical to that of $P$. miyazakii isolated from Rokuroshi in Iwakuni City, Yamaguchi Prefecture (DDBJ/EMBL/GenBank accession number: AB713405), where Sawagani was first discovered to be the second intermediate host of P. miyazakii [5].

\section{DISCUSSION}

Three lung fluke species, $P$. westermani, $P$. miyazakii and $P$. ohirai, occur in Japan and can be differentiated by the morphological features observed in living metacercariae and mounted adult fluke specimens [7, 8]. The morphological features of the metacercariae and adult worms examined in this study are consistent only with those of P. miyazakii [7, $8]$ and, thus, we identified the specimens as P. miyazakii. The ITS2 sequence data corroborate this conclusion. Therefore, Sawagani infected with P. miyazakii are concluded to still be widely prevalent in the Kawane area of Shizuoka Prefecture as reported previously [4]. Thus, the recently reported feline paragonimiasis cases [11] were likely caused by $P$. miyazakii infection. Veterinarians and owners of carnivorous companion animals, particularly cats, kept in and around the Kawane area should be aware of the risk of $P$. miyazakii infection.

Paragonimus skrjabini infections in humans and animals have been reported in China, since this species was first described in 1959 [3]. P. miyazakii was designated as a new species 2 years after the designation of $P$. skrjabini [5]. $P$. skrjabini occurs in various parts of China as well as Thailand and northeastern India [8, 14], while P. miyazakii has been reported to occur exclusively throughout most of Japan [9]. Blair et al. [2] determined that P. skrjabini found in China is very closely related to $P$. miyazakii found in Japan based on identical ITS2 sequence in a recent phylogenetic study. A subsequent study by Blair et al. [1] comparing morphometric data and molecular phylogenetic findings based on mitochondrial DNA cytochrome $c$ oxidase subunit I gene sequences showed that $P$. skrjabini from Fujian Province in Eastern China was very closely related to P. miyazakii. They proposed that these populations should be referred to as one and the same subspecies, P. skrjabini miyazakii, and that $P$. skrjabini populations from other parts of China should be referred to as a different subspecies, P. skrjabini skrjabini. Based on these findings, they concluded that $P$. skrjabini represents a species complex [1]. P. miyazakii or P. skrjabini miyazakii in Japan is a primary member of the $P$. skrjabini species complex, and it has been reported that this species/subspecies can be discriminated from P. skrjabini populations in China, even those from Fujian Province, using morphometric parameters such as the ratio of the oral sucker diameter to body length [1]. Therefore, we retain the use of the original name, P. miyazakii, in the present study. In addition, recent scientific literature using the scientific name P. miyazakii has been published, implying that this name is widely accepted [12].

In Japan, paragonimiasis is still an important foodborne helminthic zoonosis; at least 30 human cases are reported annually [10]. To establish countermeasures for controlling lung fluke infection, we previously investigated the effect of heat on the infectivity of $P$. westermani metacercariae in Sawagani. Boiling Sawagani infected with $P$. westermani metacercariae at $55^{\circ} \mathrm{C}$ for $5 \mathrm{~min}$ eliminated the infectivity to test mice [18]. However, the conditions required to reduce or eliminate the infectivity of $P$. miyazakii metacercariae harbored in Sawagani have not yet been confirmed; we are currently conducting tests on Sawagani from the Kawane area. Therefore, currently, a health education campaign that emphasizes on the risks of $P$. miyazakii infection from consuming raw and undercooked Sawagani, including those sold at retail fish markets, is recommended as the most efficient countermeasure for preventing lung fluke infections.

ACKNOWLEDGMENTS. This study was supported in part by grants for Research on Emerging and Re-emerging Infectious Diseases (H23-Shinko-Ippan-014 and H24Shinko-Ippan-006) and for Research on Food Safety (H22Shokuhin-Ippan-009) from the Ministry of Health, Labour and Welfare of Japan.

\section{REFERENCES}

1. Blair, D., Chang, Z. H., Chen, M. G., Cui, A., Wu, B., Agatsuma, T., Iwagami, M., Corlis, D., Fu, C. B. and Zhan, X. M. 2005. Paragonimus skrjabini Chen, 1959 (Digenea: Paragonimidae) and related species in eastern Asia: a combined molecular and morphological approach to identification and taxonomy. Syst. Parasitol. 60: 1-21. [Medline] [CrossRef]

2. Blair, D., Wu, B., Chang, Z. S., Gong, X., Agatsuma, T., Zhang, Y. N., Chen, S. H., Lin, J. X., Chen, M. G., Waikagul, J., Guevara, A. G., Feng, Z. and Davis, G. M. 1999. A molecular perspective on the genera Paragonimus Braun, Euparagonimus Chen and Pagumogonimus Chen. J. Helminthol. 73: 295-299. [Medline]

3. Chen, H. T. 1959. The occurrence of a new type of Paragonimus and some clinical problems related to lung flukes in China. Annual Rep. 1958, Chung Shan Med. College: 192-193 (in Chinese). 
4. Ito, J. and Mochizuki, H. 1975. Studies on the incidence of encysted larvae of Paragonimus miyazakii Kamo et al., 1961 in the crab Potamon dehaani in Shizuoka Prefecture, Japan. Jpn. J. Parasitol. 24: 241-249 (in Japanese with English abstract).

5. Kamo, H., Nishida, H., Hatsushika, R. and Tomimura, T. 1961. On the occurrence of a new lung fluke, Paragonimus miyazakii n. sp. in Japan (Trematoda: Troglotrematidae). Yonago Acta Med. 5: 43-52.

6. Kobayashi, A., Suzuki, S., Horiuchi, K., Yokogawa, M. and Araki, K. 1975. Four human cases of paragonimiasis miyazakii. Jikeikai Med. J. 22: 127-135.

7. Miyazaki, I. 1974. Lung flukes in the world. Morphology and life history. pp. 101-135. In: A Symposium on Epidemiology of Parasitic Diseases (Sasa, M. ed.), International Medical Foundation of Japan, Tokyo.

8. Miyazaki, I. 1991. Paragonimiasis. pp. 76-146. In: An Illustrated Book of Helminthic Zoonoses (Miyazaki, I. ed.), International Medical Foundation of Japan, Tokyo.

9. Nishida, H. and Shibahara, T. 2003. Epidemiology of paragonimiasis. pp. 201-217. In: Progress of Medical Parasitology in Japan, vol. 8 (Otsuru, M., Kamegai, S. and Hayashi, S. eds.), Meguro Parasitological Museum, Tokyo.

10. Obara, A., Nakamura-Uchiyama, F., Hiromatsu, K. and Nawa, Y. 2004. Paragonimiasis cases recently found among immigrants in Japan. Intern. Med. 43: 388-392. [Medline] [CrossRef]

11. Ogawa, T., Mishima, H. and Araie, T. 2011. Radiographic features of five cases of feline paragonimosis. Jpn. J. Vet. Med. Assoc. 64: 474-476 (in Japanese with English abstract).

12. Procop, G. W. 2009. North American paragonimiasis (caused by Paragonimus kellicotti) in the context of global paragonimiasis. Clin. Microbiol. Rev. 22: 415-446. [Medline] [CrossRef]

13. Shibahara, T. 1984. Studies on the lung fluke, Paragonimus westermani -diploid type-, in the northern part of Hyogo Prefecture, Japan. III. Experimental oral infection with metacercariae of rats, with reference to juvenile worms removed from the muscle. Jpn. J. Parasitol. 33: 119-132 (in Japanese with English abstract).
14. Singh, S. T., Singh, D. L. and Sugiyama, H. 2006. Possible discovery of Chinese lung fluke, Paragonimus skrjabini in Manipur, India. Southeast Asian J. Trop. Med. Public Health 37(Suppl. 3): 53-56. [Medline]

15. Sugiyama, H., Matsumoto, M., Tomimura, T. and Nishida, H. 1985. Geographical distribution of the lung fluke, Paragonimus miyazakii Kamo et al., 1961, in the southern prefectures of the Kinki District, Japan: observations on the incidence of encysted larvae of P. miyazakii in Geothelphusa dehaani in the central part of Wakayama Prefecture. Jpn. J. Vet. Sci. 47: 617-625. [Medline] [CrossRef]

16. Sugiyama, H., Morishima, Y., Kameoka, Y. and Kawanaka, M. 2002. Polymerase chain reaction (PCR)-based molecular discrimination between Paragonimus westermani and P. miyazakii at the metacercarial stage. Mol. Cell. Probes 16: 231-236. [Medline] [CrossRef]

17. Sugiyama, H., Morishima, Y., Yamasaki, H. and Kasuga, F. 2011. Detection of Paragonimus metacercariae in the Japanese freshwater crab, Geothelphusa dehaani, bought at retail fish markets, a follow-up study. Infect. Agents Surveill. Rep. 32: 172-173 (in Japanese).

18. Sugiyama, H., Morishima, Y., Yamasaki, H., Shibata, K. and Kawakami, Y. 2010. Effect of heat on the infectivity of Paragonimus metacercariae in intermediate host crabs. Clin. Parasitol. 21: 43-45 (in Japanese).

19. Sugiyama, H., Umehara, A., Morishima, Y., Yamasaki, H. and Kawanaka, M. 2009. Detection of Paragonimus metacercariae in the Japanese freshwater crab, Geothelphusa dehaani, bought at retail fish markets in Japan. Jpn. J. Infect. Dis. 62: 324-325. [Medline]

20. Yokogawa, M., Araki, K., Saito, K., Momose, T., Kimura, K., Suzuki, S., Chiba, N., Kutsumi, H. and Minai, M. 1974. Paragonimus miyazakii infections in man first found in Kanto district, Japan. Especially, on the methods of immunosero-diagnosis for paragonimiasis. Jpn. J. Parasitol. 23: 167-179 (in Japanese with English abstract). 